\title{
A longitudinal assessment of antimicrobial susceptibility among important pathogens collected as part of the Tigecycline Evaluation and Surveillance Trial (T.E.S.T.) in France between 2004 and 2012
}

Vincent Cattoir $^{1,3^{*}}$ and Michael J Dowzicky ${ }^{2}$

\begin{abstract}
Background: Clinically important Gram-positive and -negative isolates were collected from patients in France between 2004 and 2012 as a part of the Tigecycline Evaluation and Surveillance Trial.

Methods: MICs were determined using methodology described by the Clinical and Laboratory Standards Institute.

Results: In total, 17,135 isolates were contributed by 29 medical centres; respiratory (25.1\%) and cardiovascular (20.3\%) sources predominated. High susceptibility was observed among Enterococcus spp. and Staphylococcus aureus (including methicillin-resistant S. aureus [MRSA]) to linezolid (100\%), tigecycline ( $\geq 99.8 \%)$ and vancomycin $(\geq 94.6 \%)$. The percentage of MRSA decreased from 34.3\% in 2004 to 20.0\% in 2009 before increasing to 34.7\% in 2012. Vancomycin, linezolid, levofloxacin and carbapenems were highly active ( $\geq 99.6 \%)$ against Streptococcus pneumoniae; 3.2\% were PRSP. Escherichia coli showed peak susceptibility to the carbapenems ( $\geq 99.9 \%)$, tigecycline (99.3\%) and amikacin (97.9\%); significant $(p<0.01)$ decreases in susceptibility were observed for ampicillin, cefepime and ceftriaxone between 2004 and 2012. ESBL production among E. coli increased from 3.0\% (2004) to 14.9\% (2012). High susceptibility was noted among Haemophilus influenzae to levofloxacin (100\%), amoxicillin-clavulanate (99.2\%), carbapenems $(\geq 98.7 \%)$ and ceftriaxone (98.5\%); $\beta$-lactamase production fluctuated with no notable trend between 18.1\% (2007) and $27.7 \%$ (2011). Klebsiella spp. were highly susceptible to carbapenems ( $\geq 99.6 \%)$ and amikacin ( $\geq 96.4 \%) ;$ significant $(p<0.01)$ decreases in amoxicillin-clavulanate, cefepime, ceftriaxone, levofloxacin, piperacillin-tazobactam and tigecycline susceptibility were observed among K. pneumoniae between 2004 and 2012. Only imipenem was highly active (96.5\% susceptible) against Acinetobacter baumannii. Imipenem and amikacin ( $87.7 \%$ and $87.1 \%$ susceptible) were the most active agents against $P$. aeruginosa; $10.2 \%$ of isolates were categorized as multidrug resistant.
\end{abstract}

Conclusions: Carbapenems, linezolid, tigecycline and vancomycin conserved good in vitro activity against most pathogens (according to their spectrum of activity) in France between 2004 and 2012.

Keywords: France, Antimicrobial resistance, Antimicrobial surveillance, Multidrug resistance, MDR, Tigecycline

\footnotetext{
* Correspondence: cattoir-v@chu-caen.fr

${ }^{1} \mathrm{CHU}$ de Caen, Microbiologie \& Centre National de Référence de la

Résistance aux Antibiotiques (laboratoire associé Entérocoques et résistances

particulières des bactéries à Gram positif), Caen, France

${ }^{3} \mathrm{CHU}$ de Caen, Service de Microbiologie - Niveau 3, Avenue de la Côte de

Nacre-CS30001, 14033 Caen, Cedex 9, France

Full list of author information is available at the end of the article
}

() Biomed Central (c) 2014 Cattoir and Dowzicky; licensee BioMed Central Ltd. This is an Open Access article distributed under the terms of the Creative Commons Attribution License (http://creativecommons.org/licenses/by/4.0), which permits unrestricted use, distribution, and reproduction in any medium, provided the original work is properly credited. The Creative Commons Public Domain Dedication waiver (http://creativecommons.org/publicdomain/zero/1.0/) applies to the data made available in this article, unless otherwise stated. 


\section{Background}

France is home to one of the highest rates of antibiotic consumption and antimicrobial resistance in Europe [1], and has experienced rapidly changing trends of antimicrobial resistance in recent years. The European Antimicrobial Resistance Surveillance Network (EARS-Net) has reported significantly increasing levels of resistance in France [2], where $10.8 \%$ of Escherichia coli and $23.7 \%$ of Klebsiella pneumoniae isolates were reported to be intermediate or resistant to third-generation cephalosporins in 2012 (as compared with 1.9\% in 2002 and 5.1\% in 2005, respectively) [3]. Several programmes have been initiated to combat these increasing levels of resistance, including measures to control transmission of resistant pathogens, to promote the use of alcohol-based hand-rub solution in hospitals, to control/prevent the spread of emerging multidrug-resistant (MDR) organisms (i.e., vancomycinresistant enterococci [VRE], carbapenemase-producing Enterobacteriaceae) and to decrease antibiotic consumption [4]. These efforts have paid at least some dividends: declining levels of antimicrobial resistance have been reported in recent years among French isolates of Streptococcus pneumoniae to penicillin (from $36.2 \%$ in 2005 to $23.4 \%$ in 2012) and Staphylococcus aureus to methicillin (from $33.4 \%$ in 2001 to $19.1 \%$ in 2012) [3].

Tigecycline is a broad-spectrum antimicrobial agent which has been indicated for use in the treatment of complicated skin and skin structure infections (cSSTIs) and complicated intra-abdominal infections (cIAIS) (and in the USA, community-acquired bacterial pneumonia) [5]. The Tigecycline Evaluation and Surveillance Trial (T.E.S.T.) is a global surveillance study which commenced in 2004, with the intention of monitoring the activity of the broad-spectrum glycylcycline tigecycline and a panel of comparator agents against an array of clinically important Gram-positive and Gram-negative organisms. In this study, we examine the activity of tigecycline and comparators against clinically important Gram-positive and Gram-negative pathogens collected from community and nosocomial patients in France between 2004 and 2012. This manuscript serves as an update to Rodloff et al. [6], who described a collection of isolates from France, Germany, Italy, Spain and the U.K. collected as a part of T.E.S.T. between 2004 and 2006, as well as NørskovLauritsen et al. [7], who presented data on European isolates (including France) collected between 2004 and 2007.

\section{Methods}

Between 2004 and 2012 there were 29 centres in France. The majority of these centres were university hospitals. No centres contributed in all 9 study years. Three centres contributed in 8 years, two in 7 years, four in 6 years, six in 5 years, three in 4 years, three in 3 years, five in 2 years, and three in a single year.

\section{Bacterial isolates}

Each centre was required to submit a minimum of 65 Gram-positive isolates and 135 Gram-negative isolates, including at least 25 S. aureus, 15 Enterococcus spp., 15 S. pneumoniae, 10 Streptococcus agalactiae, 25 Klebsiella spp., 25 E. coli, 25 Enterobacter spp., 20 Pseudomonas aeruginosa, 15 Acinetobacter spp., $15 \mathrm{H}$. influenzae and 10 Serratia spp. isolates. Each submitted isolate had to be considered by the contributing centre to be of clinical significance as the probable causative agent of a hospitalor community-acquired infection. All body sites were considered acceptable isolate sources for this study, including body fluid, central nervous system, cardiovascular system, gastro-intestinal, genito-urinary (no more than 25\% of isolates from any centre), head, ears, eyes, nose and throat, integument, lymph, muscular, reproductive, respiratory, skeletal or medical instruments (i.e. catheters, drains, forceps, probes). No banked or stored isolates or duplicate isolates from a single patient were accepted into the T.E.S.T. study. Isolate inclusion was independent of patient age, sex, antimicrobial use and/or medical history.

All isolates were sent to a single reference laboratory, International Health Management Associates (IHMA, Schaumburg, IL), which was responsible for organism collection and transport and organism identification confirmation and development. IHMA also undertook creation and management of a centralized isolate database. Quality control (QC) checks were carried out by IHMA on approximately $10 \%$ of isolates annually.

\section{Antimicrobial susceptibility testing}

Minimum inhibitory concentrations (MICs) were determined locally using broth microdilution methodology as described by the Clinical and Laboratory Standards Institute (CLSI) [8] using either MicroScan ${ }^{\circ}$ panels (Dade Behring Inc., CA, USA) or Sensititre ${ }^{\odot}$ plates (TREK Diagnostic Systems, West Sussex, England). The test panel for the T.E.S.T. study included amikacin (AMK), amoxicillin-clavulanate (AMC), ampicillin (AMP), cefepime (CFP), ceftazidime (CTZ), ceftriaxone (CRO), imipenem (IMP), levofloxacin (LEV), linezolid (LZD), meropenem (MER), minocycline (MIN), penicillin (PEN), piperacillin-tazobactam (PTZ), tigecycline (TIG) and vancomycin (VAN). Imipenem was replaced in 2006 by meropenem due to stability issues associated with imipenem and MicroScan ${ }^{\oplus}$ panels were replaced by Sensititre ${ }^{\oplus}$ the same year. After 2006, the test panel for S. pneumoniae also included azithromycin (AZI), clarithromycin (CLA), erythromycin (ERY) and clindamycin (CLI). Clinical categorization was done using the 2013 breakpoints established by the European Committee on Antimicrobial Susceptibility Testing (EUCAST) [9]. Data are included in the tables only when interpretive breakpoints are available. 
Extended-spectrum $\beta$-lactamase (ESBL) production among E. coli and Klebsiella spp. was identified by IHMA using cefotaxime $(30 \mu \mathrm{g})$, cefotaxime-clavulanic acid $(30 / 10 \mu \mathrm{g})$, ceftazidime $(30 \mu \mathrm{g})$, and ceftazidimeclavulanic acid $(30 / 10 \mu \mathrm{g})$ discs [10]. A positive ESBL result was designated by an increase of $\geq 5 \mathrm{~mm}$ in the inhibition zone on the combination disc compared with the corresponding cephalosporin disc. Discs were manufactured by Oxoid, Inc. (Ogdensburg, NY, USA); MuellerHinton agar was produced by Remel, Inc. (Lenexa, KS, USA). $H$. influenzae isolates were tested for $\beta$-lactamase production using locally preferred methodologies. Multidrug resistance was defined as resistance to three or more classes of antimicrobial agent, and only included antimicrobials with available breakpoints. For A. baumannii antimicrobials classes (and agents) included in the analysis were aminoglycosides [AMK], carbapenems [IMP or MER], and fluoroquinolones [LEV]. For P. aeruginosa antimicrobial classes (and agents) included in the analysis were aminoglycosides [AMK], $\beta$-lactams [CFP, CTZ, PTZ], carbapenems [IMP or MER], and fluoroquinolones [LEV].

Daily QC testing was performed using QC strains Enterococcus faecalis ATCC 29212, S. aureus ATCC 29213, S. pneumoniae ATCC 49619, E. coli ATCC 25922, P. aeruginosa ATCC 27853 and $H$. influenzae ATCC 49247 and ATCC 49766, as appropriate. QC strains used for ESBL testing were K. pneumoniae ATCC 700603 (ESBLpositive) and E. coli ATCC 25922 (ESBL-negative), while $P$. aeruginosa (ATCC 27853) was used for the QC of ceftazidime and cefotaxime discs. Information on T.E.S.T. study protocols can be found online [5].

Longitudinal data were examined for statistically significant changes in susceptibility between 2004 and 2012 using the Cochran Armitage Trend Test. A positive change reflected a statistically significant decrease in susceptibility, while a negative change indicated that susceptibility had increased significantly. A $p<0.01$ was used in this analysis as a cut-off value for statistical significance (a significance value of $\mathrm{p}<0.05$ was not used here as computing a high volume of statistical tests can lead to significant results purely by chance; setting a lower significance value greatly reduces the chance of this happening).

\section{Results}

Isolates were collected from 29 centres in France between 2004 and 2012 (eight in 2004, six in 2005, 12 in 2006, 16 in 2007, 21 in 2008, 20 in 2009, 15 in 2010, five in 2011 and 23 in 2012) as a part of the T.E.S.T surveillance study.

\section{Gram-positive pathogens Enterococcus spp.}

Between 2004 and 2012, 969 isolates of E. faecalis and 332 Enterococcus faecium isolates were examined as a part of the T.E.S.T. study (Table 1). Both species were highly susceptible to linezolid (both 100\%), tigecycline (99.8\% and $100 \%$, respectively) and vancomycin $(99.3 \%$ and $94.6 \%$, respectively). E. faecalis were also highly susceptible to amoxicillin-clavulanate, ampicillin and imipenem (>96\%), while E. faecium were not ( $\leq 25 \%$ susceptible). Decreases in E. faecalis susceptibility between 2004 and 2012 to amoxicillin-clavulanate (100\% to $96.7 \%$ ) and ampicillin (100\% to $95.4 \%)$ were small but statistically significant ( $\mathrm{p}<0.01$ and $\mathrm{p}<0.001$, respectively). Of note, vancomycin resistance was observed in $0.7 \%$ of E. faecalis isolates (increasing from $0.0 \%$ in 2004 to $1.3 \%$ in 2012) and $5.4 \%$ of E. faecium isolates (increasing from $0.0 \%$ in 2004 to 4.3\% in 2012) between 2004 and 2012 in France. Linezolid and tigecycline activity were unaffected by vancomycin resistance (Table 2).

\section{S. aureus}

All $(\mathrm{N}=2229) S$. aureus isolates were susceptible to linezolid, tigecycline and vancomycin, including MRSA isolates, while $93.7 \%$ were susceptible to minocycline (Table 1). The percentage of $S$. aureus identified as MRSA in France decreased from $34.3 \%$ in 2004 to $20.0 \%$ in 2009 , but increased to $34.7 \%$ in 2012 ; the average MRSA rate over the total course of the study was $28.3 \%$ (Table 3 ). There was a statistically significant $(\mathrm{p}<0.001)$ increase in minocycline susceptibility among MRSA over the study duration (Table 2). Methicillin resistance had no impact on the activity of linezolid, minocycline, tigecycline or vancomycin.

\section{S. agalactiae}

S. agalactiae $(\mathrm{N}=859)$ were highly susceptible to most agents on the TEST panel where breakpoints exist, the notable exception being minocycline (against which only $15.4 \%$ of isolates were susceptible) (Table 1).

\section{S. pneumoniae}

S. pneumoniae $(\mathrm{N}=990)$ were highly susceptible to vancomycin (100\%), linezolid (99.9\%) and levofloxacin (99.6\%). Imipenem and meropenem were also highly active (100\% and $99.9 \%$ susceptibility, respectively), although only tested against a subset of isolates $(n=120$ and $n=$ 870) (Table 1). A $\mathrm{MIC}_{90}$ of $0.06 \mathrm{mg} / \mathrm{L}$ was reported for tigecycline (no tigecycline breakpoints are available). Statistically significant changes in susceptibility were observed between 2004 and 2012 for clindamycin (increasing from $52.3 \%$ to $67.4 \% ; \mathrm{p}<0.01$ ) and minocycline (decreasing from 55.8 to $50.3 \%$; p < 0.01) (Additional file 1: Table S1). No penicillin-resistant S. pneumoniae (PRSP) were collected in 2004 or 2011 (Table 3). The highest rate of penicillin resistance was reported in 2006 (4.6\%). The PRSP rate over the 2004-2012 period in France was $3.2 \%$ (Table 3). A statistically significant $(\mathrm{p}<0.01)$ increase in clindamycin susceptibility was 
Table 1 Minimum inhibitory concentrations $\left(\right.$ MIC $_{50}$, MIC $_{90}$, MIC range [mg/L]) and antimicrobial susceptibility (\%S) of clinically important Gram-positive and Gram-negative isolates

\begin{tabular}{llllll}
\hline Pathogen & $\mathrm{N}$ & $\mathrm{MIC}_{50}$ & $\mathrm{MIC}_{90}$ & MIC Range & $\% \mathrm{~S}$ \\
\hline Gram-positive & & & &
\end{tabular}

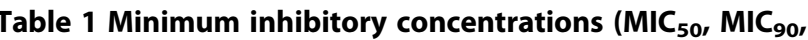
MIC range [mg/L]) and antimicrobial susceptibility (\%S) of clinically important Gram-positive and Gram-negative isolates (Continued)

E. faecalis

AMC

AMP

IMP

LZD

$\mathrm{TIG}$

VAN

E. faecium

$\mathrm{AMC}$

AMP

IMP

LZD

TIG

VAN

S. aureus

LEV

LZD

MIN

PEN

TIG

VAN

S. agalactiae

LEV

LZD

MIN

PEN

TIG

VAN

S. pneumoniae

AMP

AZI

CRO

CLA

CLI

ERY

IMP

LEV

LZD

MER

MIN

$\begin{array}{ccc}969 & 0.5 & 1 \\ 969 & 1 & 2 \\ 137 & 1 & 4 \\ 969 & 2 & 2 \\ 969 & 0.12 & 0.25 \\ 969 & 1 & 2\end{array}$

$\begin{array}{cc}\leq 0.03-\geq 16 & 99.1 \\ \leq 0.06-\geq 32 & 98.8 \\ \leq 0.12-16 & 96.4 \\ \leq 0.5-4 & 100 \\ \leq 0.008-0.5 & 99.8 \\ 0.25-\geq 64 & 99.3\end{array}$

PEN

VAN

$\begin{array}{cc}990 & \leq 0.06 \\ 990 & 0.25\end{array}$

Gram-negative

E. aerogenes $0.25-\geq 64$

99.3

AMK

CFP

CRO

IMP

LEV

MER

PTZ

TIG

\section{E. cloacae}

AMK

CFP

CRO

IMP

LEV

MER

$$
\leq 0.5-4
$$

$\leq 0.25-\geq 16$

$\leq 0.06-\geq 16$

93.7

PTZ

TGC

E. coli

AMK

AMC

AMP

CFP

CRO

IMP

LEV

MER

PTZ

TGC

H. influenzae

AMC

AMP

CRO

IMP

LEV

MER

MIN

K. oxytoca

AMK

$\begin{array}{ccccc}561 & 2 & 8 & \leq 0.5-64 & 95.9 \\ 561 & \leq 0.5 & 2 & \leq 0.5-\geq 64 & 87.0 \\ 561 & 0.5 & 32 & \leq 0.06-\geq 128 & 56.7 \\ 81 & 0.5 & 1 & \leq 0.06-4 & 97.5 \\ 561 & 0.06 & \geq 16 & \leq 0.008-\geq 16 & 76.5 \\ 480 & \leq 0.06 & 0.12 & \leq 0.06-8 & 98.3 \\ 561 & 8 & 64 & 0.25-\geq 256 & 59.5 \\ 561 & 0.5 & 2 & 0.12-16 & 87.0\end{array}$

$\begin{array}{ccccc}1665 & 1 & 4 & \leq 0.5-\geq 128 & 96.7 \\ 1665 & \leq 0.5 & 8 & \leq 0.5-\geq 64 & 66.5 \\ 1665 & 1 & \geq 128 & \leq 0.06-\geq 128 & 50.7 \\ 226 & 0.5 & 1 & \leq 0.06-8 & 99.1 \\ 1665 & 0.06 & \geq 16 & \leq 0.008-\geq 16 & 73.1 \\ 1439 & \leq 0.06 & 0.25 & \leq 0.06-\geq 32 & 99.4 \\ 1665 & 4 & \geq 256 & \leq 0.06-\geq 256 & 59.3 \\ 1665 & 0.5 & 2 & 0.06-16 & 85.0 \\ & & & & \\ 2284 & 2 & 4 & \leq 0.5-\geq 128 & 97.9 \\ 2284 & 8 & 32 & 0.25-\geq 64 & 70.8 \\ 2284 & \geq 64 & \geq 64 & \leq 0.5-\geq 64 & 38.4 \\ 2284 & \leq 0.5 & 8 & \leq 0.5-\geq 64 & 84.3 \\ 2284 & \leq 0.06 & 64 & \leq 0.06-\geq 128 & 84.0 \\ 324 & 0.25 & 0.5 & \leq 0.06-2 & 100 \\ 2284 & 0.03 & \geq 16 & \leq 0.008-\geq 16 & 79.9 \\ 1960 & \leq 0.06 & \leq 0.06 & \leq 0.06-4 & 99.9 \\ 2284 & 2 & 16 & \leq 0.06-\geq 256 & 89.0 \\ 2284 & 0.25 & 0.5 & \leq 0.008-2 & 99.3\end{array}$

$1191 \quad 0.5$

$1191 \leq 0.5$

1191

$\leq 0.06$

0.25

0.015

$\leq 0.06$

$\leq 0.5$

$\leq 0.12-\geq 32$

99.9

$\leq 0.25-\geq 16$

47.6

$$
\begin{array}{r}
1 \\
32 \\
\leq 0.06 \\
0.5 \\
0.015 \\
0.12 \\
1
\end{array}
$$

$\leq 0.12-16$

$\leq 0.5-\geq 64$

$\leq 0.06-4 \quad 98.5$

$\leq 0.06-4 \quad 98.7$

$\leq 0.008-1 \quad 100$

$\leq 0.06-0.5 \quad 100$

$\leq 0.5-16 \quad 90.8$

$\leq 0.5-\geq 128$

98.7 
Table 1 Minimum inhibitory concentrations $\left(\mathrm{MIC}_{50}, \mathrm{MIC}_{90}\right.$, MIC range $[\mathrm{mg} / \mathrm{L}])$ and antimicrobial susceptibility (\%S) of clinically important Gram-positive and Gram-negative isolates (Continued)

\begin{tabular}{|c|c|c|c|c|c|}
\hline AMC & 695 & 2 & 32 & $0.25-\geq 64$ & 79.7 \\
\hline CFP & 695 & $\leq 0.5$ & 2 & $\leq 0.5-\geq 64$ & 89.2 \\
\hline CRO & 695 & $\leq 0.06$ & 8 & $\leq 0.06-\geq 128$ & 83.3 \\
\hline IMP & 102 & 0.25 & 0.5 & $\leq 0.06-1$ & 100 \\
\hline LEV & 695 & 0.06 & 1 & $\leq 0.008-\geq 16$ & 90.5 \\
\hline MER & 593 & $\leq 0.06$ & $\leq 0.06$ & $\leq 0.06-\geq 32$ & 99.7 \\
\hline PTZ & 695 & 2 & $\geq 256$ & $\leq 0.06-\geq 256$ & 83.3 \\
\hline TGC & 695 & 0.25 & 1 & $0.015-8$ & 95.4 \\
\hline \multicolumn{6}{|c|}{ K. pneumoniae } \\
\hline AMK & 1524 & 1 & 4 & $\leq 0.5-\geq 128$ & 96.4 \\
\hline AMC & 1524 & 4 & 32 & $0.5-\geq 64$ & 72.6 \\
\hline CFP & 1524 & $\leq 0.5$ & 32 & $\leq 0.5-\geq 64$ & 79.4 \\
\hline CRO & 1524 & $\leq 0.06$ & $\geq 128$ & $\leq 0.06-\geq 128$ & 77.2 \\
\hline IMP & 211 & 0.25 & 0.5 & $\leq 0.06-2$ & 100 \\
\hline LEV & 1524 & 0.06 & 8 & $\leq 0.008-\geq 16$ & 82.2 \\
\hline MER & 1313 & $\leq 0.06$ & $\leq 0.06$ & $\leq 0.06-\geq 32$ & 99.6 \\
\hline PTZ & 1524 & 2 & 64 & $0.12-\geq 256$ & 81.3 \\
\hline TGC & 1524 & 0.5 & 2 & $0.06-16$ & 87.6 \\
\hline \multicolumn{6}{|c|}{ S. marcescens } \\
\hline AMK & 895 & 2 & 4 & $\leq 0.5-\geq 128$ & 97.1 \\
\hline CFP & 895 & $\leq 0.5$ & 1 & $\leq 0.5-\geq 64$ & 94.4 \\
\hline CRO & 895 & 0.25 & 8 & $\leq 0.06-\geq 128$ & 80.4 \\
\hline IMP & 118 & 0.5 & 1 & $\leq 0.06-4$ & 96.6 \\
\hline LEV & 895 & 0.12 & 2 & $0.015-\geq 16$ & 87.2 \\
\hline MER & 777 & $\leq 0.06$ & 0.12 & $\leq 0.06-\geq 32$ & 98.7 \\
\hline PTZ & 895 & 2 & 16 & $\leq 0.06-\geq 256$ & 88.7 \\
\hline TGC & 895 & 1 & 2 & $0.015-8$ & 80.1 \\
\hline \multicolumn{6}{|c|}{ A. baumannii } \\
\hline AMK & 1161 & 4 & 64 & $\leq 0.5-\geq 128$ & 75.6 \\
\hline IMP & 170 & 0.5 & 2 & $\leq 0.06-\geq 32$ & 96.5 \\
\hline LEV & 1161 & 0.25 & 8 & $\leq 0.008-\geq 16$ & 56.8 \\
\hline MER & 991 & 0.5 & 8 & $\leq 0.06-\geq 32$ & 83.7 \\
\hline \multicolumn{6}{|c|}{ P. aeruginosa } \\
\hline AMK & 1780 & 4 & 16 & $\leq 0.5-\geq 128$ & 87.1 \\
\hline CFP & 1780 & 4 & 32 & $\leq 0.5-\geq 64$ & 77.5 \\
\hline$C T Z$ & 1780 & $\leq 8$ & 32 & $\leq 8-\geq 64$ & 75.6 \\
\hline IMP & 260 & 1 & 8 & $0.12-\geq 32$ & 87.7 \\
\hline LEV & 1780 & 1 & $\geq 16$ & $\leq 0.008-\geq 16$ & 58.3 \\
\hline MER & 1520 & 0.5 & 8 & $\leq 0.06-\geq 32$ & 75.4 \\
\hline PTZ & 1780 & 8 & $\geq 256$ & $0.12-\geq 256$ & 72.5 \\
\hline
\end{tabular}

AMK, amikacin; AMC, amoxicillin-clavulanate; AMP, ampicillin; CFP, cefepime; $C T Z$, ceftazidime; CRO, ceftriaxone; IMP, imipenem; LEV, levofloxacin; LZD, linezolid; MER, meropenem; MIN, minocycline; PEN, penicillin; PTZ, piperacillin-tazobactam; TIG, tigecycline; VAN, vancomycin. observed among PRSP isolates (Table 2). Susceptibly to levofloxacin, linezolid, meropenem, and vancomycin were largely unaffected by penicillin resistance (Table 2). The $\mathrm{MIC}_{90}$ for tigecycline was $0.03 \mathrm{mg} / \mathrm{L}$ against penicillinresistant isolates.

\section{Gram-negative pathogens \\ Enterobacter spp.}

Meropenem, imipenem and amikacin were the most active agents against Enterobacter spp., with 98.3\% $(\mathrm{n}=480)$, 97.5\% $(\mathrm{n}=81)$ and $95.9 \%$ of E. aerogenes $(\mathrm{N}=561)$ and 99.4\% ( $\mathrm{n}=1439), 99.1 \%(\mathrm{n}=226)$ and $96.7 \%$ of E. cloacae $(\mathrm{N}=1665)$ isolates susceptible, respectively (Table 1). E. aerogenes and E. cloacae were $87.0 \%$ and $85.0 \%$ susceptible to tigecycline, respectively. No statistically significant changes in susceptibility over time were reported for Enterobacter spp..

\section{E. coli}

E. coli $(\mathrm{N}=2284)$ were highly susceptible to imipenem (100\%; $\mathrm{n}=324)$, meropenem (99.9\%; $\mathrm{n}=1960)$, tigecycline (99.3\%) and amikacin (97.9\%). Statistically significant decreases in susceptibility were observed to ampicillin ( $<<$ $0.001 ; 55.4 \%$ to $33.2 \%$ ), cefepime ( $<<0.0001 ; 97.0 \%$ to $81.7 \%)$ and ceftriaxone ( $<<0.0001 ; 96.0 \%$ to $81.1 \%)$ between 2004 and 2012 (Additional file 1: Table S1). The percentage of ESBL-positive $E$. coli isolates increased from $3.0 \%$ in 2004 to $14.9 \%$ in 2012 , reaching a maximum of $17.5 \%$ in 2009 (Table 3). Statistically significant increases in susceptibility were observed among ESBL-positive $E$. coli to amikacin $(\mathrm{p}<0.001)$, amoxicillin-clavulanate $(\mathrm{p}<$ $0.001)$, levofloxacin $(\mathrm{p}<0.01)$ and piperacillin-tazobactam $(\mathrm{p}<0.01)$ (Table 2). Carbapenem and tigecycline activity were not impacted by ESBL production (Table 2).

\section{H. influenzae}

All isolates of $H$. influenzae ( $\mathrm{N}=1191)$ were susceptible to levofloxacin and meropenem ( $\mathrm{n}=1035)$; susceptibility was also high to amoxicillin-clavulanate (99.2\%), imipenem $(98.7 \% ; \mathrm{n}=156)$ and ceftriaxone $(98.5 \%)$. The $\mathrm{MIC}_{90}$ of tigecycline was $0.25 \mathrm{mg} / \mathrm{L}$. The percentage of $\beta$-lactamase positive isolates did not change notably between 2004 and 2012 (Table 3). As expected, the in vitro activity of ampicillin was dramatically reduced against $\beta$-lactamasepositive $H$. influenzae (Table 2).

\section{Klebsiella spp.}

Both $K$. oxytoca $(\mathrm{N}=695)$ and $K$. pneumoniae $(\mathrm{N}=1524)$ were fully susceptible to imipenem $(\mathrm{n}=102$ and 211, respectively). High levels of susceptibility were also reported for meropenem (99.7\% $[n=593]$ and 99.6\% [ $n=1313]$, respectively) and amikacin (98.7\% and $96.4 \%$, respectively) (Table 1). Statistically significant decreases in susceptibility were observed among $K$. pneumoniae to amoxicillin- 
Table 2 MIC $_{90}$ (mg/L), antimicrobial susceptibility (\%S) and statistically significant changes in susceptibility among resistant pathogen phenotypes

\begin{tabular}{|c|c|c|c|c|}
\hline Pathogen & Antimicrobial & $\mathrm{MIC}_{90}$ & $\% \mathrm{~S}$ & Significance $^{\mathrm{a}}$ \\
\hline \multicolumn{5}{|l|}{ Gram-positive } \\
\hline \multirow{5}{*}{$\begin{array}{l}\text { E. faecium, VRE } \\
(n=18[0 / 18])\end{array}$} & AMC & $\geq 16$ & 16.7 & N.S. \\
\hline & AMP & $\geq 32$ & 16.7 & N.S. \\
\hline & LZD & 2 & 100 & - \\
\hline & $\mathrm{TIG}$ & 0.25 & 100 & - \\
\hline & VAN & $\geq 64$ & 0.0 & - \\
\hline \multirow{6}{*}{$\begin{array}{l}\text { S. aureus, MRSA } \\
(n=631[77 / 554])\end{array}$} & LEV & 32 & 13.2 & N.S. \\
\hline & LZD & 2 & 100 & - \\
\hline & MIN & 0.5 & 93.5 & $p<0.001(-)$ \\
\hline & PEN & $\geq 16$ & 0.0 & - \\
\hline & $\mathrm{TIG}$ & 0.25 & 100 & - \\
\hline & VAN & 1 & 100 & - \\
\hline \multirow{12}{*}{$\begin{array}{l}\text { S. pneumoniae, PRSP } \\
\left(\mathrm{n}=32 ; 31^{\mathrm{b}}\right)\end{array}$} & AMP & 8 & 0.0 & - \\
\hline & AZI & $\geq 128$ & 19.4 & N.S. \\
\hline & $\mathrm{CRO}$ & 2 & 6.3 & N.S. \\
\hline & CLA & $\geq 128$ & 19.4 & N.S. \\
\hline & $\mathrm{CLI}$ & $\geq 128$ & 32.3 & $p<0.01(-)$ \\
\hline & ERY & $\geq 128$ & 19.4 & N.S. \\
\hline & LEV & 2 & 96.9 & N.S. \\
\hline & LZD & 1 & 100 & - \\
\hline & MER & 1 & 96.9 & N.S. \\
\hline & $\mathrm{MIN}$ & $\geq 16$ & 18.8 & N.S. \\
\hline & PEN & 4 & 0.0 & - \\
\hline & VAN & 0.5 & 100 & - \\
\hline
\end{tabular}

Gram-negative

E. coli, ESBL $(n=275[17 / 258])$

$\begin{array}{lcccc} & \text { AMC } & 32 & 36.7 & p<0.001(-) \\ & \text { AMP } & \geq 64 & 0.0 & - \\ & \text { CFP } & \geq 64 & 4.7 & \text { N.S. } \\ & \text { CRO } & \geq 128 & 0.0 & - \\ & \text { IMP } & 0.5 & 100 & - \\ & \text { LEV } & \geq 16 & 37.8 & p<0.01(-) \\ \text { H. influenzae, BL-POS } & \text { MER } & \leq 0.06 & 100 & - \\ (\mathrm{n}=269[32 / 237]) & \text { PTZ } & 64 & 72.4 & p<0.01(-) \\ & \text { TIG } & 0.5 & 98.9 & \text { N.S. } \\ & \text { AMP } & 2 & 98.1 & \text { N.S. } \\ & \text { CRO } & \leq 64 & 0.4 & \text { N.S. } \\ & \text { IMP } & 1 & 100 & -\end{array}$

Table 2 MIC $_{90}$ (mg/L), antimicrobial susceptibility (\%S) and statistically significant changes in susceptibility among resistant pathogen phenotypes (Continued)

\begin{tabular}{lcccc}
\hline & LEV & 0.015 & 100 & - \\
& MER & 0.12 & 100 & - \\
& MIN & 1 & 92.6 & N.S. \\
$\begin{array}{l}\text { K. pneumoniae, ESBL } \\
(n=274[19 / 255])\end{array}$ & AMK & 16 & 85.0 & N.S. \\
& AMC & 32 & 16.1 & N.S. \\
& CFP & $\geq 64$ & 7.3 & N.S. \\
& CRO & $\geq 128$ & 1.8 & N.S. \\
& IMP & 0.5 & 100 & N.S. \\
& LEV & $\geq 16$ & 29.6 & N.S. \\
& MER & 0.12 & 98.4 & - \\
& PTZ & $\geq 256$ & 39.4 & N.S. \\
& TIG & 2 & 78.1 & N.S.
\end{tabular}

${ }^{a} A$ negative (-) change in significance indicates an increase in susceptibility; N.S., not significant. A cut-off of $p<0.1$ was used for statistical significance testing.

Values given in square parentheses refer to the number of isolates tested against imipenem and meropenem, respectively (and, where different, ampicillin [ $\left.{ }^{\mathrm{b}}\right]$ ). Only seven vancomycin-resistant $E$. faecalis were collected during this study; data not presented.

clavulanate ( $\mathrm{p}<0.0001 ; 84.8 \%$ to $69.5 \%)$, cefepime ( $\mathrm{p}<$ $0.0001 ; 95.5 \%$ to $69.9 \%)$, ceftriaxone $(\mathrm{p}<0.0001 ; 90.9 \%$ to $69.9 \%)$, levofloxacin $(\mathrm{p}<0.0001 ; 93.9 \%$ to $77.4 \%)$, piperacillin-tazobactam $(\mathrm{p}<0.0001 ; 95.5 \%$ to $82.4 \%)$ and tigecycline $(\mathrm{p}<0.01 ; 93.9 \%$ to $84.9 \%)$ over the 2004-2012 interval (Additional file 1: Table S1). ESBL production among $K$. pneumoniae isolates increased from $7.6 \%$ in 2004 to $23.0 \%$ in 2012 (Table 3). Carbapenem activity was not impacted by ESBL production, while amikacin and tigecycline activity decreased by approximately $10 \%$ (Table 2 ). No statistically significant changes in susceptibility were reported for $K$. oxytoca.

\section{S. marcescens}

The most active antimicrobial agents in this study against $S$. marcescens $(\mathrm{N}=895)$ were meropenem $(98.7 \%$ susceptible; $\mathrm{n}=777)$, amikacin (97.1\% susceptible), imipenem $(96.6 \%$ susceptible; $\mathrm{n}=118)$ and cefepime $(94.4 \%$ susceptible). No statistically significant changes in susceptibility over time were reported.

\section{A. baumannii}

The most active agent against $A$. baumannii $(\mathrm{N}=1161)$ was imipenem (96.5\% susceptible; $\mathrm{n}=170)$, although data are only available up to 2007 (Table 1). No breakpoint is available for tigecycline, for which a $\mathrm{MIC}_{90}$ of $1 \mathrm{mg} / \mathrm{L}$ was recorded. Multidrug resistance was reported among $4.7 \%$ of $A$. baumannii isolates between 2004 and 2012, reaching a maximum of $6.7 \%$ in 2010 (Table 3). 
Table 3 Percentages of resistant phenotypes among Gram-positive and Gram-negative isolates by year, 2004-2012

\begin{tabular}{|c|c|c|c|c|c|c|c|c|c|c|c|}
\hline Pathogen & $\mathbf{N}$ & $\begin{array}{l}2004 \\
\text { n (\%) }\end{array}$ & $\begin{array}{l}2005 \\
\text { n (\%) }\end{array}$ & $\begin{array}{l}2006 \\
\text { n (\%) }\end{array}$ & $\begin{array}{l}2007 \\
\text { n (\%) }\end{array}$ & $\begin{array}{l}2008 \\
\text { n (\%) }\end{array}$ & $\begin{array}{l}2009 \\
\text { n (\%) }\end{array}$ & $\begin{array}{l}2010 \\
\text { n (\%) }\end{array}$ & $\begin{array}{l}2011 \\
\text { n (\%) }\end{array}$ & $\begin{array}{l}2012 \\
\text { n (\%) }\end{array}$ & $\begin{array}{c}\text { 2004-12 } \\
\text { n (\%) }\end{array}$ \\
\hline \multicolumn{12}{|l|}{ Gram-positive } \\
\hline E. faecalis, VRE & 969 & $0(0.0)$ & $0(0.0)$ & $1(1.5)$ & $0(0.0)$ & $0(0.0)$ & $2(1.1)$ & $1(0.7)$ & $1(3.8)$ & $2(1.3)$ & $7(0.7)$ \\
\hline E. faecium, VRE & 332 & $0(0.0)$ & $0(0.0)$ & $0(0.0)$ & $2(4.9)$ & $8(10.8)$ & $2(3.3)$ & $3(5.5)$ & $1(6.7)$ & $2(4.3)$ & $18(5.4)$ \\
\hline S. aureus, MRSA & 2229 & $34(34.3)$ & $20(31.7)$ & $40(30.5)$ & $88(29.6)$ & $135(28.2)$ & $77(20.0)$ & $75(26.1)$ & $15(23.4)$ & $147(34.7)$ & $631(28.3)$ \\
\hline S. pneumoniae, PRSP & 990 & $0(0.0)$ & $1(3.4)$ & $3(4.6)$ & $3(2.0)$ & $9(4.5)$ & $7(4.0)$ & $6(4.1)$ & $0(0.0)$ & $3(2.0)$ & $32(3.2)$ \\
\hline \multicolumn{12}{|l|}{ Gram-negative } \\
\hline A. baumannii, MDR & 1161 & $0(0.0)$ & $0(0.0)$ & $2(2.0)$ & $7(4.9)$ & $12(5.2)$ & $12(5.2)$ & $13(6.7)$ & $1(3.0)$ & $7(4.7)$ & $54(4.7)$ \\
\hline E. coli, ESBL & 2284 & $3(3.0)$ & $2(3.7)$ & $8(4.6)$ & $18(6.5)$ & $58(11.8)$ & 75 (17.5) & $46(13.9)$ & $13(16.9)$ & $52(14.9)$ & $275(12.0)$ \\
\hline H. influenzae, BL-Pos & 1191 & $13(23.2)$ & $6(23.1)$ & $17(27.4)$ & $30(18.1)$ & $53(21.1)$ & $56(25.0)$ & $36(23.7)$ & $13(27.7)$ & $45(21.7)$ & 269 (22.6) \\
\hline K. pneumoniae, ESBL & 1524 & $5(7.6)$ & $5(9.3)$ & $9(10.3)$ & $20(12.3)$ & 64 (18.6) & $47(16.4)$ & $58(24.9)$ & $11(22.0)$ & $55(23.0)$ & 274 (18.0) \\
\hline P. aeruginosa, MDR & 1780 & $2(2.5)$ & $0(0.0)$ & 17 (11.2) & $23(10.7)$ & $39(10.2)$ & $37(11.0)$ & $33(12.5)$ & $9(14.8)$ & $21(8.1)$ & $181(10.2)$ \\
\hline
\end{tabular}

ESBL, extended-spectrum $\beta$-lactamase; BL-Pos, $\beta$-lactamase-positive; MDR, multidrug-resistant; MRSA, methicillin-resistant S. aureus; PRSP, penicillin-resistant S. pneumoniae; VRE, vancomycin-resistant Enterococcus.

Results do not exactly match those presented by Nørskov-Lauritsen et al. [7] due to subsequent addition and deletion of isolates from the T.E.S.T. database.

\section{P. aeruginosa}

Imipenem $(\mathrm{n}=260)$ and amikacin were the most active agents against $P$. aeruginosa with $87.7 \%$ and $87.1 \%$ susceptibility, respectively (Table 1 ). A total of $10.2 \%$ of $P$. aeruginosa isolates were MDR, ranging from $0.0 \%$ in 2005 to $14.8 \%$ in 2011 (Table 3).

\section{Discussion}

This report updates data previously presented by Rodloff et al. [6] for France (as well as Germany, Italy, Spain and the U.K.) between 2004 and 2006 and Nørskov-Lauritsen et al. [7] for data collected between 2004 and 2007. The data described in their reports are included in the dataset described in this manuscript. Susceptibility results are difficult to compare between these two earlier reports and the current study as CLSI interpretive breakpoints were used in Rodloff et al. [6] and Nørskov-Lauritsen et al. [7] while EUCAST breakpoints have been used in the current manuscript. No vancomycin-resistant enterococci were reported in either earlier study in France; however, small percentages of vancomycin-resistant E. faecalis $(0.7 \%)$ and E. faecium (5.4\%) were collected in the current study. As the data show, the majority of vancomycin-resistant enterococci were collected during or after 2008 (three isolates were collected in 2006 and 2007 but were not reported by Rodloff et al. [6] and Nørskov-Lauritsen et al. [7] as they were entered into the database after the data cut-offs for these publications). Rates of MRSA were comparable between the three reports $(28.3 \%$ in the current study, $28.3 \%$ in Rodloff et al. [6], and 31.5\% in NørskovLauritsen et al. [7]); however, the rate of penicillinresistant S. pneumoniae was lower in the current study when compared with Nørskov-Lauritsen et al. [7] (3.2\% and $16.8 \%$, respectively). No S. pneumoniae data was presented by Rodloff et al. [6]. This difference is likely due in part to the use of CLSI breakpoints by Nørskov-Lauritsen et al. (resistance breakpoint $\geq 2 \mathrm{mg} / \mathrm{L}$, compared to $\geq 4$ $\mathrm{mg} / \mathrm{L}$ used by EUCAST); the removal of $236 \mathrm{~S}$. pneumoniae isolates from the T.E.S.T. database whose MICs could not be verified (i.e., isolates which could not be revived for retesting or which died on transport from the contributing centre to IHMA) may have also influenced this PRSP difference.

ESBL production among E. coli and $K$. pneumoniae was higher in the current study; $12.0 \%$ and $18.0 \%$ compared with $4.9 \%$ and $9.5 \%$ and $5.1 \%$ and $9.8 \%$ in Rodloff et al. [6] and Nørskov-Lauritsen et al. [7], respectively. As rates of ESBLs were higher in the later years of this study (2008 onwards) this difference is not unexpected. Rates of multidrug-resistant $A$. baumannii and $\beta$-lactamase producing $H$. influenzae were similar between the current report and Nørskov-Lauritsen et al. [7]. (approximately $5 \%$ and $22 \%$, respectively), although the definition of MDR A. baumannii in Nørskov-Lauritsen et al. [7]. also included cephalosporins. Data on multidrug-resistant $A$. baumannii and $H$. influenzae were not reported by Rodloff et al. [6]. As the isolates presented by Rodloff et al. [6] and Nørskov-Lauritsen et al. [7] are also included in this report comparisons between these three reported must be treated with some caution. However, the increases in rates of vancomycin-resistant enterococci, and ESBL-producing E. coli and $K$. pneumoniae are cause for concern and warrant further monitoring.

One factor that could influence the difference in resistance rates between the reports is the presence of centre specific outbreaks. Outbreaks of resistant pathogens have been described in several medical centres in France in recent years, caused by carbapenemase-producing [11] or metallo- $\beta$-lactamase-producing $K$. pneumoniae [12], MDR A. baumannii [13], glycopeptide-intermediate $S$. aureus 
[14] and vancomycin-resistant enterococci $[4,15]$. These outbreaks were controlled with infection control measures, including strict enforcement of hygiene precautions, limiting transfer of patients to other wards, isolating infected patients with dedicated staff and the closure of infected wards. These outbreaks of highly resistant pathogens reinforce the clinical importance of antimicrobial agents such as tigecycline, daptomycin, linezolid, and vancomycin, which often retain excellent in vitro activity against even highly resistant pathogens $[16,17]$.

As a result of a resistance control programme started in 2003 in 38 French teaching hospitals, vancomycinresistant enterococci and carbapenemase-producing Enterobacteriaceae cases were controlled while MRSA incidence declined by two thirds; however, a dramatic increase in the percentage of ESBL-positive Enterobacteriaceae was noted [4]. Similarly, a long-term study involving 933 health care facilities carried out by the French national healthcare-associated infection early-warning, investigation and surveillance network (RAISIN) led to a $43 \%$ decrease in MRSA while ESBL-positive Enterobacteriaceae increased by $182 \%$ [18]. The epidemiology of ESBLproducing pathogens can be very complex [19], and ESBL-positive Enterobacteriaceae are increasing in prevalence so rapidly that they may soon become the most widespread MDR pathogens in French hospitals [20]. ESBL levels among $E$. coli and $K$. pneumoniae increased markedly over the course of the T.E.S.T. study; however, MRSA levels in the current study decreased between 2004 (34.3\%) and 2009 (20.0\%) but increased from 2011 (23.4\%) to 2012 (34.7\%). This increase in MRSA levels was unexpected and may have been due to regional factors such as localised outbreak(s) of resistant isolates.

In a review of data collected by the Pneumococcus Surveillance Network (PSN) in France in 2007, Kempf et al. [21] reported a PRSP percentage of $6.6 \%$ among S. pneumoniae isolates collected from adults and children. This PRSP occurrence is twice that recorded in the current manuscript for France between 2004 and 2012, and three times higher than the value reported in T.E.S.T. for 2007 alone. This difference is due in part to Kempf et al. [21] using a resistance breakpoint of $>1 \mathrm{mg} / \mathrm{L}$ for penicillin, compared with $\geq 4 \mathrm{mg} / \mathrm{L}$ used in this T.E.S.T. study. Sizeable $(>20 \%)$ regional variations in the prevalence of penicillin-non-susceptible $S$. pneumoniae and a high number of isolates collected from children $(27.9 \%)$ were also reported by Kempf et al. [21].

Tigecycline and linezolid demonstrated good activity against the Gram-positive isolates in this study. In the case of enterococci the activity of tigecycline and linezolid has also been demonstrated by others [15,22,23]. Bourdon et al. [15] performed susceptibility testing on $602 \mathrm{E}$. faecium and $30 \mathrm{E}$. faecalis isolates, all VRE, collected from 112 French hospitals between 2006 and 2008 and observed $100 \%$ susceptibility to tigecycline and linezolid. Similarly, Marcadé et al. [22] described seven glycopeptideresistant $E$. faecium isolates from a single hospital in Paris which possessed both $v a n A$ and $\operatorname{vanB}$ resistance genes; all were susceptible to tigecycline and linezolid, as well as daptomycin. Bérenger et al. [23] examined 60 glycopeptide-resistant, epidemiologically unrelated clinical isolates of E. faecium collected in France between 2006 and 2008; all were susceptible to linezolid while 59 were tigecycline-susceptible (the remaining isolate had intermediate susceptibility for tigecycline).

In the current T.E.S.T. report, the levels of $\beta$-lactamase positive isolates of $H$. influenzae fluctuated year-on-year (between 18.1\% and 27.7\%) between 2004 and 2012, with no discernible pattern over time. A statistically significant decrease in the occurrence of $\beta$-lactamase-positive, ampicillin-resistant isolates among non-typeable $H$. influenzae was reported in France between 2001 and 2008 [24], with the rate decreasing from $35.6 \%$ in $2001-02$ to $13.5 \%$ in 2007-08; however, this study only included isolates collected from patients $\leq 5$ years in age.

\section{Conclusions}

Programmes aimed at controlling and/or reducing the prevalence of drug-resistant pathogens in France have been successful against some important pathogens, such as MRSA and VRE, but other resistant pathogens continue to increase in prevalence across the country, most notably ESBL-positive Enterobacteriaceae. These trends highlight the importance of surveillance studies such as T.E.S.T., which monitor pathogen resistance rates against key antimicrobial agents both nationally and globally. Tigecycline possesses good in vitro activity against many resistant pathogens, including ESBL producers, and thus could be a useful tool in the treatment of resistant infections in France in the future.

\section{Additional file}

Additional file 1: Table S1. MIC 90 (mg/L) and antimicrobial susceptibility (\%) of clinically important Gram-positive and Gram-negative isolates.

\section{Competing interests \\ VC has no competing interests to declare. \\ MJD is an employee of Pfizer, Inc.}

\section{Authors' contributions}

VC was involved in the collection and interpretation of data used in this study as well as the drafting and editing of this manuscript, and has given approval for this manuscript to be published. MJD was involved conception and design of the T.E.S.T. surveillance study as well as the drafting and editing of this manuscript and has given approval for this manuscript to be published. Both authors have read and approved this final manuscript draft.

\section{Acknowledgements}

The authors wish to acknowledge and thank all T.E.S.T. investigators and laboratories for their participation in this study, as well as the staff at IHMA for their coordination of T.E.S.T. This study was sponsored by Pfizer Inc. 
No authors were paid for their contributions to this manuscript. Medical writing support was provided by Dr. Rod Taylor at Micron Research Ltd, Ely, UK and was funded by Pfizer Inc. Micron Research Ltd also provided data management services which were funded by Pfizer Inc.

\section{Author details}

${ }^{1} \mathrm{CHU}$ de Caen, Microbiologie \& Centre National de Référence de la Résistance aux Antibiotiques (laboratoire associé Entérocoques et résistances particulières des bactéries à Gram positif), Caen, France. ${ }^{2}$ Pfizer Inc Collegeville, PA, USA. ${ }^{3} \mathrm{CHU}$ de Caen, Service de Microbiologie - Niveau 3, Avenue de la Côte de Nacre-CS30001, 14033 Caen, Cedex 9, France.

Received: 14 April 2014 Accepted: 3 November 2014

Published: 1 December 2014

\section{References}

1. Dumartin $C$, L'Hériteau F, Péfau $M$, Bertrand $X$, Jarno $P$, Boussat $S$, Angora $P$, Lacavé L, Saby K, Savey A, Nguyen F, Carbonne A, Rogues AM: Antibiotic use in 530 French hospitals: results from a surveillance network at hospital and ward levels in 2007. J Antimicrob Chemother 2010, 65:2028-2036.

2. European Centre for Disease Prevention and Control: Antimicrobial resistance surveillance in Europe 2012. Annual Report of the European Antimicrobial Resistance Surveillance Network (EARS-Net). Stockholm: ECDC; 2013.

3. European Centre for Disease Prevention and Control: Antimicrobial resistance interactive database (EARS-Net). [http://www.ecdc.europa.eu/ en/healthtopics/antimicrobial_resistance/database/Pages/database.aspx]

4. Fournier S, Brun-Buisson C, Jarlier V: Twenty years of antimicrobial resistance control programme in a regional multi hospital institution, with focus on emerging bacteria (VRE and CPE). Antimicrob Resist Infect Control 2012, 13:1-9.

5. Pfizer Inc. (Wyeth Pharmaceuticals): Tygacil ${ }^{\circledR}$ Product Insert. http://www. pfizerpro.com/hcp/tygacil.

6. Rodloff AC, Leclercq R, Debbia EA, Cantón R, Oppenheim BA, Dowzicky MJ: Comparative analysis of antimicrobial susceptibility among organisms from France, Germany, Italy, Spain and the UK as part of the tigecycline evaluation and surveillance trial. Clin Microbiol Infect 2008, 14:307-314.

7. Nørskov-Lauritsen N, Marchandin H, Dowzicky MJ: Antimicrobial susceptibility of tigecycline and comparators against bacterial isolates collected as part of the TEST study in Europe (2004-2007). Int J Antimicrob Agents 2009, 34:121-130.

8. Clinical and Laboratory Standards Institute (CLSI): Methods for Dilution Antimicrobial Susceptibility Tests for Bacteria That Grow Aerobically, Approved Standard. 8th ed. Document M7-A8. Wayne, PA: CLSI; 2009.

9. European Committee on Antimicrobial Susceptibility Testing: Clinical breakpoints. [http://www.eucast.org/clinical_breakpoints/]

10. Clinical and Laboratory Standards Institute (CLSI): Performance Standards for Antimicrobial Susceptibility Testing: 23rd ed. Document M100-S23. Wayne, PA: CLSI; 2013.

11. Kassis-Chikhani N, Saliba F, Carbonne A, Neuville S, Decre D, Sengelin C, Guerin C, Gastiaburu N, Lavigne-Kriaa A, Boutelier C, Arlet G, Samuel D, Castaing D, Dussaix E, Jarlier V: Extended measures for controlling an outbreak of VIM-1 producing imipenem-resistant Klebsiella pneumoniae in a liver transplant centre in France, 2003-2004. Euro Surveill 2010, 15.

12. Carbonne A, Thiolet JM, Fournier S, Fortineau N, Kassis-Chikhani N, Boytchev I, Aggoune M, Seguier JC, Senechal H, Tavolacci MP, Coignard B, Astagneau P, Jarlier V: Control of a multi-hospital outbreak of KPC-producing Klebsiella pneumoniae type 2 in France, September to October 2009. Euro Surveill 2010, 15

13. Ayraud-Thévenot S, Huart C, Mimoz O, Taouqi M, Laland C, Bousseau A, Castel O: Control of multi-drug-resistant Acinetobacter baumannii outbreaks in an intensive care unit: feasibility and economic impact of rapid unit closure. J Hosp Infect 2012, 82:290-292.

14. Parer $\mathrm{S}$, Lotthé $\mathrm{A}$, Chardon $\mathrm{P}$, Poncet $\mathrm{R}$, Jean-Pierre $\mathrm{H}$, Jumas-Bilak E: An outbreak of heterogeneous glycopeptide-intermediate Staphylococcus aureus related to a device source in an intensive care unit. Infect Control Hosp Epidemiol 2012, 33:167-174.

15. Bourdon N, Fines-Guyon M, Thiolet JM, Maugat S, Coignard B, Leclercq R, Cattoir V: Changing trends in vancomycin-resistant enterococci in French hospitals, 2001-08. J Antimicrob Chemother 2011, 66:713-721.

16. Kalil AC, Klompas M, Haynatzki G, Rupp ME: Treatment of hospital-acquired pneumonia with linezolid or vancomycin: a systematic review and meta-analysis. BMJ Open 2013, 3:e003912.
17. Lee YT, Tsao SM, Hsueh PR: Clinical outcomes of tigecycline alone or in combination with other antimicrobial agents for the treatment of patients with healthcare-associated multidrug-resistant Acinetobacter baumannii infections. Eur J Clin Microbiol Infect Dis 2013, 32:1211-1220.

18. Carbonne A, Arnaud I, Maugat S, Marty N, Dumartin C, Bertrand X, Bajolet O, Savey A, Fosse T, Eveillard M, Sénéchal H, Coignard B, Astagneau P, Jarlier V, MDRB Surveillance National Steering Group (BMR-Raisin): National multidrug-resistant bacteria (MDRB) surveillance in France through the RAISIN network: a 9 year experience. J Antimicrob Chemother 2013, 68:954-959.

19. Marcadé G, Brisse S, Bialek S, Marcon E, Leflon-Guibout V, Passet V, Moreau R, Nicolas-Chanoine $\mathrm{MH}$ : The emergence of multidrug-resistant Klebsiella pneumoniae of international clones ST13, ST16, ST35, ST48 and ST101 in a teaching hospital in the Paris region. Epidemiol Infect 2013, 141:1705-1712.

20. Gbaguidi-Haore H, Dumartin C, L'Hériteau F, Péfau M, Hocquet D, Rogues AM, Bertrand X, ATB-RAISIN Network Steering Committee: Antibiotics involved in the occurrence of antibiotic-resistant bacteria: a nationwide multilevel study suggests differences within antibiotic classes. J Antimicrob Chemother 2013, 68:461-470.

21. Kempf M, Baraduc R, Bonnabau H, Brun M, Chabanon G, Chardon H, Croizé J, Demachy MC, Donnio PY, Dupont P, Fosse T, Gibel L, Gravet A, Grignon B, Hadou T, Hamdad F, Joly-Guillou ML, Koeck JL, Maugein J, Péchinot A, Ploy MC, Raymond J, Ros A, Roussel-Delvallez M, Segonds C, Vergnaud M, Vernet-Garnier V, Lepoutre A, Gutmann L, Varon E, et al: Epidemiology and antimicrobial resistance of Streptococcus pneumoniae in France in 2007: data from the pneumococcus surveillance network. Microb Drug Resist 2011, 17:31-36.

22. Marcadé G, Micol JB, Jacquier H, Raskine L, Donay JL, Nicolas-Viaud $\mathrm{S}$, Rouveau M, Ribaud P, Dombret H, Leclercq R, Cambau E: Outbreak in a haematology unit involving an unusual strain of glycopeptide-resistant Enterococcus faecium carrying both vanA and vanB genes. J Antimicrob Chemother 2014, 69:500-505

23. Bérenger $R$, Bourdon $N$, Auzou $M$, Leclerca $R$, Cattoir V: In vitro activity of new antimicrobial agents against glycopeptide-resistant Enterococcus faecium clinical isolates from France between 2006 and 2008. Med Mal Infect 2011, 41:405-409.

24. Dabernat $\mathrm{H}$, Delmas C: Epidemiology and evolution of antibiotic resistance of Haemophilus influenzae in children 5 years of age or less in France, 2001-2008: a retrospective database analysis. Eur J Clin Microbiol Infect Dis 2012, 31:2745-2753.

doi:10.1186/2047-2994-3-36

Cite this article as: Cattoir and Dowzicky: A longitudinal assessment of antimicrobial susceptibility among important pathogens collected as part of the Tigecycline Evaluation and Surveillance Trial (T.E.S.T.) in France between 2004 and 2012. Antimicrobial Resistance and Infection Control 2014 3:36.

\section{Submit your next manuscript to BioMed Central and take full advantage of:}

- Convenient online submission

- Thorough peer review

- No space constraints or color figure charges

- Immediate publication on acceptance

- Inclusion in PubMed, CAS, Scopus and Google Scholar

- Research which is freely available for redistribution 